\title{
Construction of a new technique in Aitken extrapolation method for solving Fredholm integral equation of the first kind with iterated kernel.
}

\author{
Aml Jassim Mohammed \& Junaid Idrees Mustafa \\ Department of Mathematics / College of Education \\ University of Mosul \\ Received \\ Accepted \\ 23/4/2007 \\ 17/07/2007
}

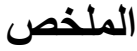

في هذا البحث تم استخدام تكنيك جديد لطريقة اتكن لحل معادلة فريدهولم التكامليه من

النوع الاول. هذه الطريقه تحتاج الى خطوات تكراريه لذلك, أعتمدت على الطريقة التكرارية التي

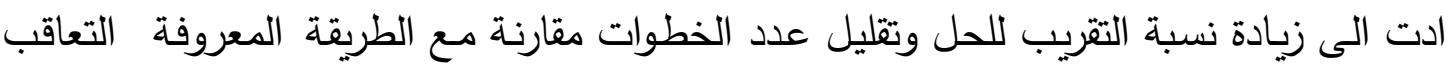

والطريقة التكرارية نفسها.
\end{abstract}

Abstract

In this paper a new technique for Aitken extrapolation method is used to solve F.I.E. $1^{s t} \mathrm{k}$. This method requires many reiterative steps. Therefore, it depends on the iterative method which results in an increase in the rate of approximation of the solution and simultaneously it minimizes the number of the steps in comparison with the outdated successive method and iterative method itself.

\section{Introduction :}

The problem considered here is that of quickly numerically solving Fredholm integral equation of the form:

$h(x) u(x)=f(x)+\lambda \int_{a}^{b} k(x, y) u(y) d y$

in this study, it is assumed that $f(x)$ is a continuous function and $k(x, y)$ are continuous for $a \leq x \leq b, a \leq y \leq b$. The function $f(x), k(x, y)$ and the parameter $\lambda$ are assumed to be given ,where as $x$ and $y$ are real variables. When $h(x)=0$ we will obtain:

$$
f(x)=\lambda \int_{a}^{b} k(x, y) u(y) d y
$$

this is called Fredholm integral equation of the first kind (F.I.E $\left.1^{s t} \mathrm{k}\right)$. 
In 1979 Gerald was used Aitken extrapolation. In 1999 Sulaiman used Aitken extrapolation on successive method to solve F.I.E. ${ }^{\text {nd }} \mathrm{k}$. And in 2004 it was used Aitken Extrapolation on Adomian method to solve F.I.E $2^{\text {nd }} \mathrm{k}$.

In this study, a new technique on Aitken extrapolation method is used to treat the solution of F.I.E. ${ }^{\text {st }} \mathrm{k}$.

\section{Definition Iterated kernel [ 4 ]}

Equation (2) can be modified as $f(x)=\lambda \sum_{i=0}^{\infty} \psi_{i}(x)$ where the function $\psi_{i}(x)$ may be found from the formulas:

$$
\begin{aligned}
& \psi_{1}(x)=\int_{a}^{b} k(x, y) f(y) d y \\
& \psi_{2}(x)=\int_{a}^{b} k(x, y) \psi_{1}(y) d y=\int_{a}^{b} k_{2}(x, y) f(y) d y \\
& \cdot \\
& \psi_{n}(x)=\int_{a}^{b} k(x, y) \psi_{n-1}(y) d y=\int_{a}^{b} k_{n}(x, y) f(y) d y
\end{aligned}
$$

here

$$
\begin{aligned}
& k_{1}(x, y)=k(x, y) \\
& k_{2}(x, y)=\int_{a}^{b} k(x, z) k_{1}(z, y) d z \\
& k_{n}(x, y)=\int_{a}^{b} k(x, z) k_{n-1}(z, y) d z
\end{aligned}
$$




\section{Iterative Method to Solve F.I.E. $1^{s t}$.k.[ 4 ]}

The integral equation (2) with $\lambda=1$ will obtain

$$
f(x)=\int_{a}^{b} k(x, y) u(y) d y
$$

Let $u_{0}(x)=f(x)$ continuous function in $[\mathrm{a}, \mathrm{b}]$ is an initial approximate solution of $u(x)$, put the continuous function $u_{0}(x)$ into the right side of equation (3) we get:

$$
u_{1}(x)=\int_{a}^{b} k(x, y) u_{0}(y) d y
$$

where $u_{1}(x)$ is a first approximation of $u(x)$. Thus, the defined function $u_{1}(x)$ is also continuous in [a,b], and in continuing this process we obtain a sequence of functions $u_{0}(x), u_{1}(x), \ldots . ., u_{n}(x)$ where

$$
u_{i}(x)=\int_{a}^{b} k(x, y) u_{i-1}(y) d y \quad i=1,2, \ldots
$$

The sum of these functions approximate to the exact solution.

\section{Method of Successive Approximation [ 4,2]}

This method is used for solving F.I.E. ${ }^{s t} \mathrm{k}$. starting with substituting zero approximation $u_{0}(x)$ in the integral of equation (3) to obtain a first approximation $u_{1}(x)$,

$$
f(x)=\int_{a}^{b} k(x, y) u_{0}(y) d y \quad a \leq y \leq b
$$

this process can be continued to obtain the $\mathrm{n}$-th approximation:

$$
f(x)=\int_{a}^{b} k(x, y) u_{n-1}(y) d y
$$

equation (7) is the approximation solution to the exact solution $u(x)$

\section{Aitken Extrapolation Method [ 3 ]}

From three successive approximations of the solution $u_{0}(x), u_{1}(x)$ and $u_{2}(x)$ we extrapolate to an important estimate. This can be done by considering the following formula: 


$$
\Phi(x)=\frac{u_{0}(x) u_{2}(x)-u_{1}^{2}(x)}{u_{2}(x)-2 u_{1}(x)+u_{0}(x)}
$$

\section{A new Technique for Aitken Formula}

This technique uses iteration method and simple changing to equation (8) to solve F.I.E. $1^{\text {st }} \mathrm{k}$.

$$
\delta(x)=\frac{3 u_{0}(x) u_{2}(x)-u^{2}(x)}{u_{2}(x)-2 u_{1}(x)+u_{0}(x)}
$$

Without this changing, the solution can not solve F.I.E. $1^{\text {st }} \mathrm{k}$ by Aitken extrapolation only.

\section{Numerical Examples and Results}

Example (1) consider the integral equation:

$$
f(x)=2 \int_{0}^{1} k(x, y) u(y) d y
$$

where $\quad k(x, y)=x^{2} y, \quad f(x)=\frac{x^{2}}{2}$ with the exact solution $u(x)=x^{2}$

Sol Apply the iterative method

$$
\begin{aligned}
& u_{0}(x)=f(x)=\frac{x^{2}}{2} \\
& u_{1}(x)=2 \int_{a}^{b} k(x, y) u_{0}(y) d y=2 \int_{0}^{1} x^{2} y \frac{y^{2}}{2} d y=\frac{x^{2}}{4} \\
& u_{2}(x)=2 \int_{a}^{b} k(x, y) u_{1}(y) d y=2 \int_{0}^{1} x^{2} y \frac{y^{2}}{4} d y=\frac{x^{2}}{8}
\end{aligned}
$$

these three approximate solutions can be substituting in the following equation : 


$$
\begin{aligned}
& \delta(x)=\frac{3 u_{0}(x) u_{2}(x)-u^{2}{ }_{1}(x)}{u_{2}(x)-2 u_{1}(x)+u_{0}(x)} \\
& \delta(x)=\frac{3 \frac{x^{2}}{2} \frac{x^{2}}{8}-\left(\frac{x^{2}}{4}\right)^{2}}{\frac{x^{2}}{8}-2 \frac{x^{2}}{4}+\frac{x^{2}}{2}} \\
& \delta(x)=x^{2},
\end{aligned}
$$

which is the exact solution of the above example.

In table 1 the comparison between iterative method, successive method and a new technique method depends on least square error.

Table 1

\begin{tabular}{|c|c|c|c|c|}
\hline $\mathbf{X}$ & $\begin{array}{c}\text { Exact } \\
x^{2}\end{array}$ & $\begin{array}{c}\text { Iterative } \\
7 x^{2}\end{array}$ & $\begin{array}{c}\text { Successive } \\
x^{2}\end{array}$ & New technique \\
& & $\frac{x^{2}}{8}$ & \\
\hline $\mathbf{0 . 0}$ & $\mathbf{0 . 0 0 0 0 0}$ & $\mathbf{0 . 0 0 0 0 0}$ & $\mathbf{0 . 0 0 0 0 0}$ & $\mathbf{0 . 0 0 0 0 0}$ \\
\hline $\mathbf{0 . 1}$ & $\mathbf{0 . 0 1 0 0 0}$ & $\mathbf{0 . 0 0 8 8 0}$ & $\mathbf{0 . 0 1 2 5 0}$ & $\mathbf{0 . 0 1 0 0 0}$ \\
\hline $\mathbf{0 . 2}$ & $\mathbf{0 . 0 4 0 0 0}$ & $\mathbf{0 . 0 3 5 0 0}$ & $\mathbf{0 . 0 0 5 0 0}$ & $\mathbf{0 . 0 4 0 0 0}$ \\
\hline $\mathbf{0 . 3}$ & $\mathbf{0 . 0 9 0 0 0}$ & $\mathbf{0 . 0 7 8 7 0}$ & $\mathbf{0 . 0 1 1 2 5}$ & $\mathbf{0 . 0 9 0 0 0}$ \\
\hline $\mathbf{0 . 4}$ & $\mathbf{0 . 1 6 0 0 0}$ & $\mathbf{0 . 1 4 0 0 0}$ & $\mathbf{0 . 0 2 0 0 0}$ & $\mathbf{0 . 1 6 0 0 0}$ \\
\hline $\mathbf{0 . 5}$ & $\mathbf{0 . 2 5 0 0 0}$ & $\mathbf{0 . 2 1 8 7 5}$ & $\mathbf{0 . 0 3 1 2 5}$ & $\mathbf{0 . 2 5 0 0 0}$ \\
\hline $\mathbf{0 . 6}$ & $\mathbf{0 . 3 6 0 0 0}$ & $\mathbf{0 . 3 1 5 0 0}$ & $\mathbf{0 . 0 4 5 0 0}$ & $\mathbf{0 . 3 6 0 0 0}$ \\
\hline $\mathbf{0 . 7}$ & $\mathbf{0 . 4 9 0 0 0}$ & $\mathbf{0 . 4 2 8 7 5}$ & $\mathbf{0 . 0 6 1 2 5}$ & $\mathbf{0 . 4 9 0 0 0}$ \\
\hline $\mathbf{0 . 8}$ & $\mathbf{0 . 6 4 0 0 0}$ & $\mathbf{0 . 5 6 0 0 0}$ & $\mathbf{0 . 0 8 0 0 0}$ & $\mathbf{0 . 6 4 0 0 0}$ \\
\hline $\mathbf{0 . 9}$ & $\mathbf{0 . 8 1 0 0 0}$ & $\mathbf{0 . 7 0 8 7 5}$ & $\mathbf{0 . 1 0 1 2 5}$ & $\mathbf{0 . 8 1 0 0 0}$ \\
\hline $\mathbf{1}$ & $\mathbf{1 . 0 0 0 0 0}$ & $\mathbf{0 . 8 7 5 0 0}$ & $\mathbf{0 . 1 2 5 0 0}$ & $\mathbf{1 . 0 0 0 0 0}$ \\
\hline & L.S.E & $\mathbf{0 . 0 3 9 5 8}$ & $\mathbf{1 . 9 3 9 5 6}$ & $\mathbf{0 . 0 0 0 0 0}$ \\
\hline
\end{tabular}

Example (2) consider the integral equation:

$$
f(x)=\int_{0}^{\frac{\pi}{2}} k(x, y) u(y) d y \text { where }
$$

$$
\begin{aligned}
& k(x, y)=\sin (x) \cos (y) \quad, f(x)=\frac{\sin (x)}{6} \text { and the exact solution is } \\
& u(x)=\frac{\sin (x)}{3} .
\end{aligned}
$$


Sol Apply the iterative method

$$
\begin{aligned}
& u_{0}(x)=f(x)=\frac{\sin (x)}{6} \\
& u_{1}(x)=\int_{a}^{b} k(x, y) u_{0}(y) d y=\int_{0}^{\frac{\pi}{2}} \sin (x) \cos (y) \frac{\sin (y)}{6} d y=\frac{\sin (x)}{12} \\
& u_{2}(x)=\int_{a}^{b} k(x, y) u_{1}(y) d y=\int_{0}^{\frac{\pi}{2}} \sin (x) \cos (y) \frac{\sin (y)}{12} d y=\frac{\sin (x)}{24}
\end{aligned}
$$

these three approximate solutions can be substituting in the following equation :

$$
\begin{aligned}
& \delta(x)=\frac{3 u_{0}(x) u_{2}(x)-u^{2}{ }_{1}(x)}{u_{2}(x)-2 u_{1}(x)+u_{0}(x)} \\
& \delta(x)=\frac{3 \frac{\sin (x)}{6} \frac{\sin (x)}{24}-\left(\frac{\sin (x)}{12}\right)^{2}}{\frac{\sin (x)}{24}-2 \frac{\sin (x)}{12}+\frac{\sin (x)}{6}} \\
& \delta(x)=\frac{\sin (x)}{3},
\end{aligned}
$$

which is the exact solution of the above example.

In table 2 the comparison between iterative method, successive method and new technique method depends on least square error. 


\begin{tabular}{|c|c|c|c|c|}
\hline \multicolumn{5}{c|}{ Table 2 } \\
\hline $\mathrm{X}$ & $\begin{array}{c}\text { Exact } \\
\sin (x) \\
3\end{array}$ & $\begin{array}{c}\text { Iterative } \\
7 \sin (x) \\
24\end{array}$ & $\begin{array}{c}\text { Successive } \\
\frac{\sin (x)}{24}\end{array}$ & New technique \\
\hline 0 & 0.00000 & 0.00000 & 0.00000 & 0.00000 \\
\hline$\pi / 20$ & 0.05214 & 0.04563 & 0.00652 & 0.05214 \\
\hline $2 \pi / 20$ & 0.10306 & 0.09013 & 0.01288 & 0.10306 \\
\hline $3 \pi / 20$ & 0.15133 & 0.13241 & 0.01816 & 0.15133 \\
\hline $4 \pi / 20$ & 0.19593 & 0.17144 & 0.02449 & 0.19593 \\
\hline $5 \pi / 20$ & 0.23570 & 0.20624 & 0.02946 & 0.23570 \\
\hline $6 \pi / 20$ & 0.26972 & 0.23596 & 0.03371 & 0.26972 \\
\hline $7 \pi / 20$ & 0.29760 & 0.25988 & 0.03713 & 0.29760 \\
\hline $8 \pi / 20$ & 0.31702 & 0.27739 & 0.03963 & 0.31702 \\
\hline $9 \pi / 20$ & 0.32923 & 0.28808 & 0.04115 & 0.32923 \\
\hline$\pi / 2$ & 0.33333 & 0.29166 & 0.04167 & 0.33333 \\
\hline \multicolumn{2}{|c|}{ L.S.E } & 0.00955 & 0.46788 & $3.0815-33$ \\
\hline
\end{tabular}

\section{Conclusion:}

1) Aitken extrapolation formula uses successive approximation method. So, we can use iterative method by depending on Aitken formula.

2) We can find the solution of the F.I.E. $1^{s t} \mathrm{k}$. by iterative method only, but many steps are used to get a good approximate solution.

3) There is a problem to solve F.I.E. $1^{s t} \mathrm{k}$. by using Aitken formula by depending on iterative method, Therefore, a new technique for Aitken formula helps to solve this problem in applying few steps.

\section{References}

[1] Amal, J. M., (2004), “ Approximate Solution for Solving Fredholm Integral Equations of the Second Kind ", M.Sc., Thesis, AL-Mustansiriya University.

[2] Green .C.D., (1969) , “ Integral Equation Methods”, Lecturer in the Department of Mathematics University of Dundee.

[3] Gerald, C.F, and Wheathley, P.O; (1979), "Applied Numerical Analysis" Adssdison Wesley printed in the United State of American.

[4] Jerri, A. J., (1985) ,'Introduction to Integral Equations with Applications", Marcel Dekker, Inc. New Yourk and Basel.

[5] Sulaiman, N.A., (1999), " New Techniques in the Numerical Solutions of Fredholm and Voltera Integral Equations", Ph.D., Thesis, Salahaddin University. 